\title{
NOTE
}

1 B. Béri • S. J. Hogan • G. Stépán

\section{Structural stability of a light rotating beam under combined loads}

Received: 20 December 2016 / Revised: 14 April 2017

(C) Springer-Verlag GmbH Austria 2017

\begin{abstract}
This paper investigates the structural stability of long boring or milling tools. The tool is modelled by a rotating cantilever beam that is subject to compression and torsion, manifested by semi-tangential torque. The three-dimensional mathematical model is based on Euler-Bernoulli beam theory considering a linear threedimensional problem. We obtain a dimensionless relationship between the relative importance of rotation, compression, and torsion that reveals the stability boundaries of the system.
\end{abstract}

\section{Modelling}

Consider a long boring or a milling tool (see Fig. 1a) modelled by a straight vertical cantilever beam. The beam rotates about its vertical axis as well as being subjected to torsion $M_{t}$ and compression $m g$. Due to the presence of torsion, we are not able to analyse the system in two-dimensions [1]. The compression can be modelled by a lumped mass $m$ attached to the free end of the beam (see Fig. 2a) that is much larger than the mass of the beam. Thus, the mass of the beam might be neglected. The beam is considered to be prismatic, homogeneous, linearly elastic and inextensible. It is either in compression or in tension depending on whether it stands upward or downward, respectively. The described system might become unstable depending upon the speed of rotation, the compression, the torsion, or a combination of all three [1].

The arrangement of the model and the corresponding notation can be seen in Fig. 2a where the gravitational acceleration is denoted by $\mathbf{g}$, the angular velocity is $\omega$, the centrifugal force is $m \omega^{2} \mathbf{d}_{1}$, the compression is $m \mathbf{g}$ and the torsional moment vector is $\mathbf{M}_{t}$. Note that the twisting moment is assumed to be semi-tangential $[3,4]$ depicted in Fig. 1b, that is, the forces $F$ acting on the beam generate an axial torque $\mathbf{M}_{t}$ that is able to tilt about both the $y$ and $z$ axes. By taking into account only small displacement $\mathbf{r}=\operatorname{col}(v w)$ and angles $\psi, \theta$ during buckling, the linearised form of the torque is $\mathbf{M}_{t}=M_{t} \operatorname{col}\left(1 \delta_{v}^{\prime} / 2 \delta_{w}^{\prime} / 2\right)$ where $M_{t}=4 F a$, and the bending components of $\mathbf{M}_{t}$ come from its resolution with respect to the principal system $(\xi, \eta, \zeta)$ and by using the definition of the semi-tangential torque in the sense of Ziegler [3] (see Fig. 1b, c). In case of the principal system, we consider $\xi$ to be tangential to the deflected beam, while $\eta$ is parallel to the $(x-y)$ plane and $\zeta$ is parallel to the $(x-z)$ plane. The notations $\delta_{v}^{\prime}$ and $\delta_{w}^{\prime}$ define the corresponding angular rotations of the end of the beam (see Fig. 2a).

B. Béri $(\bowtie) \cdot$ G. Stépán

Department of Applied Mechanics, Budapest University of Technology and Economics, Budapest, Hungary

E-mail: beri.bence@gmail.com

G. Stépán

E-mail: stepan@mm.bme.hu

S. J. Hogan

Department of Engineering Mathematics, University of Bristol, Bristol, UK

E-mail: s.j.hogan@bristol.ac.uk 


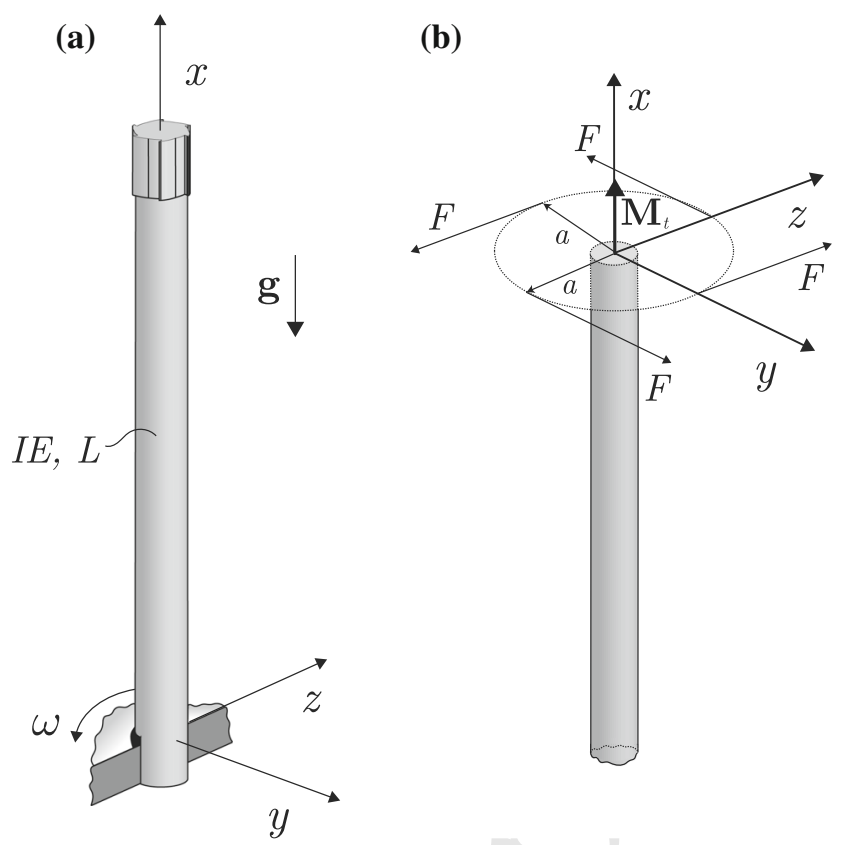

(c)

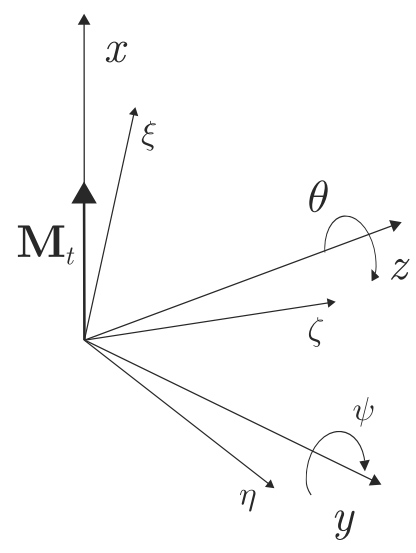

Fig. 1 a Model of the rotating tool. b Concept of the semi-tangential moment in the sense of Ziegler [3]. $\mathbf{c}$ Formulation of torsion

The mathematical model is obtained using the Euler-Bernoulli connection between curvature and bending moment [6]

$$
\frac{\partial \theta(\tilde{s})}{\partial \tilde{s}}=-\frac{M_{z}(\tilde{s})}{I E}, \quad \frac{\partial \psi(\tilde{s})}{\partial \tilde{s}}=-\frac{M_{y}(\tilde{s})}{I E},
$$

where the slope angles in the direction $y$ and $z$ are defined by $\theta$ and $\psi$ (see Fig. 2b), respectively, and $\tilde{s}$ is the arc length coordinate. The bending moment functions $M_{y, z}(\tilde{s})$ are expressed about $y$ axis and $z$ axis as follows:

$$
\begin{aligned}
& M_{y}(\tilde{s})=-M_{t} \theta(\tilde{s})+m g \tilde{s} \psi(\tilde{s})-m g \delta_{w}+m \omega^{2} \delta_{w}(\tilde{s}-L)+\frac{1}{2} M_{t} \delta_{v}^{\prime}, \\
& M_{z}(\tilde{s})=M_{t} \psi(\tilde{s})+m g \tilde{s} \theta(\tilde{s})-m g \delta_{v}+m \omega^{2} \delta_{v}(\tilde{s}-L)-\frac{1}{2} M_{t} \delta_{w}^{\prime},
\end{aligned}
$$

where $\delta_{v}=v+\delta v$ and $\delta_{w}=w+\delta w$ at the end of the beam (see Fig. 2b). Since the variation of the torsional moment (the projection of $\mathbf{M}_{t}$ to $\xi$ ) is of second order, the torsional stiffness of the beam is irrelevant from the viewpoint of buckling [6].

The dimensionless length coordinate $s=\tilde{s} / L$ is normalised by the length $L$ of the beam. By taking into account Eqs. (1) and (2), and neglecting the nonlinear terms of the bending functions above, the bending moment balance gives the dimensionless differential equation system $[2,5,6]$ for small $\theta$ and $\psi$ in the form

$$
\begin{gathered}
\frac{\partial^{2} \theta(s)}{\partial s^{2}}+\alpha \frac{\partial \psi(s)}{\partial s}+\gamma \theta(s)+\chi \delta_{v}=0, \\
\frac{\partial^{2} \psi(s)}{\partial s^{2}}-\alpha \frac{\partial \theta(s)}{\partial s}+\gamma \psi(s)+\chi \delta_{w}=0,
\end{gathered}
$$

where the parameter $\alpha=M_{t} L / I E$ specifies the relative importance of torsion to bending stiffness, $\gamma=$ $m g L^{2} / I E$ is the relative importance of gravity to bending stiffness and $\chi=m \omega^{2} L^{3} / I E$ is the relative importance of rotation to bending stiffness.

Let us introduce a complex function $d(s)=\theta(s)+\mathrm{i} \psi(s)$. Hence from Eq. (3), we have

$$
\frac{\partial^{2} d(s)}{\partial s^{2}}-\mathrm{i} \alpha \frac{\partial d(s)}{\partial s}+\gamma d(s)+\chi d_{1}=0
$$




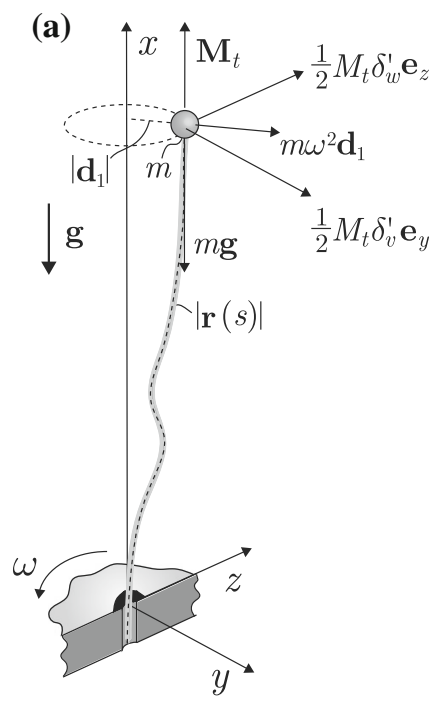

(b)

Fig. 2 a Rotating beam subjected to torsion and compression. b Infinitesimal piece of the beam

where $d_{1}=\delta_{v}+\mathrm{i} \delta_{w}$ and $\mathbf{d}_{1}=\operatorname{col}\left(\delta_{v} \delta_{w}\right)$. The boundary conditions are

$$
\theta(0)=0,\left.\quad \frac{\partial \theta(s)}{\partial s}\right|_{s=1}=-\frac{1}{2} \alpha \delta_{w}^{\prime},
$$

$$
\psi(0)=0,\left.\quad \frac{\partial \psi(s)}{\partial s}\right|_{s=1}=\frac{1}{2} \alpha \delta_{v}^{\prime},
$$

where the free end conditions are originated in the semi-tangential torque [3] as discussed above. Using the complex function $d$, the boundary conditions above can be expressed as

$$
d(0)=0,\left.\quad \frac{\partial d(s)}{\partial s}\right|_{s=1}=\frac{1}{2} \mathrm{i} \alpha d_{1}^{\prime},
$$

where $d_{1}^{\prime}=\delta_{v}^{\prime}+\mathrm{i} \delta_{w}^{\prime}$.

In addition, we are able to specify a linear connection between an assumed radial function $r(s)$ and $d(s)$ (see Fig. 2b)

$$
\frac{\partial r(s)}{\partial s}=d(s),
$$

where $r(s)=v(s)+\mathrm{i} w(s)$. It provides three additional conditions

$$
r(0)=0, \quad r(1)=d_{1},\left.\quad \frac{\partial r(s)}{\partial s}\right|_{s=1}=d_{1}^{\prime},
$$

for the complex form Eq. (4) when it is transformed for $r(s)$.

\section{Stability}

The general solution of Eq. (4) is given by

$$
\begin{gathered}
d(s)=A e^{\lambda_{1} s}+B e^{\lambda_{2} s}-\frac{\chi}{\gamma} d_{1}, \quad \lambda_{1,2}=(\mu \pm v) \mathrm{i}, \\
\mu=\alpha / 2, \quad v=\sqrt{\alpha^{2}+4 \gamma} / 2,
\end{gathered}
$$


where $\lambda_{1,2}$ are purely imaginary eigenvalues. Applying Eq. (6), the unknown coefficients $A, B$ are given by

$$
A=\frac{\chi}{\gamma} d_{1}-\frac{\frac{1}{2} \mathrm{i} \alpha d_{1}^{\prime}-\frac{\chi}{\gamma} d_{1} \lambda_{1} e^{\lambda_{1}}}{\lambda_{2} e^{\lambda_{2}}-\lambda_{1} e^{\lambda_{1}}}, \quad B=\frac{\frac{1}{2} \mathrm{i} \alpha d_{1}^{\prime}-\frac{\chi}{\gamma} d_{1} \lambda_{1} e^{\lambda_{1}}}{\lambda_{2} e^{\lambda_{2}}-\lambda_{1} e^{\lambda_{1}}} .
$$

In view of Eq. (7), the radial function $r(s)$ is then given by

$$
r(s)=A \frac{e^{\lambda_{1} s}}{\lambda_{1}}+B \frac{e^{\lambda_{2} s}}{\lambda_{2}}-\frac{\chi}{\gamma} d_{1} s+C .
$$

By means of Eq. (8), we could eliminate $d_{1}$ and $d_{1}^{\prime}$, to find

$$
\frac{\chi}{\gamma}=\frac{\frac{e^{\lambda_{2}}\left(\mathrm{i} \alpha-2 \lambda_{2}\right)-e^{\lambda_{1}}\left(\mathrm{i} \alpha-2 \lambda_{1}\right)}{\mathrm{i} \alpha\left(\frac{1-e^{\lambda_{1}}}{\lambda_{1}}+1\right)\left(e^{\lambda_{1}}-e^{\lambda_{2}}\right)}}{1+\frac{\lambda_{2}\left(1-e^{\lambda_{1}}\right)-\lambda_{1}\left(1-e^{\lambda_{2}}\right)}{\lambda_{1} \lambda_{2}\left(\frac{1-e^{\lambda_{1}}}{\lambda_{1}}+1\right)\left(\frac{e^{\lambda_{1}}-e^{\lambda_{2}}}{1-e^{\lambda_{1}}}\right)}+\frac{\lambda_{2}^{2} e^{\lambda_{2}}\left(1-e^{\lambda_{1}}+\lambda_{1}\right)-\lambda_{1}^{2} e^{\lambda_{1}}\left(1-e^{\lambda_{2}}+\lambda_{2}\right)}{\frac{\mathrm{i} \alpha \lambda_{1} \lambda_{2}}{2}\left(\frac{1-e^{\lambda_{1}}}{\lambda_{1}}+1\right)\left(e^{\lambda_{1}}-e^{\lambda_{2}}\right)} .}
$$

In order to proceed, we note that the right-hand side of Eq. (12) is in the form

$$
\frac{\chi}{\gamma}=\frac{P_{r}+\mathrm{i} P_{i}}{Q_{r}+\mathrm{i} Q_{i}}
$$

where $P_{r}, P_{i}$ and $Q_{r}, Q_{i}$ are real quantities. Now the left-hand side of Eq. (12) is real. So the right-hand side of Eq. (13) must also be real and so its imaginary part has to vanish. Hence

$$
P_{i} Q_{r}-P_{r} Q_{i}=0
$$

But then the real part of the right-hand side of Eq. (13) can be simplified so that we find

$$
\frac{\chi}{\gamma}=\frac{P_{r}}{Q_{r}}
$$

So using Eqs. (15) and (12) simplifies greatly to become

$$
\chi=-\frac{\gamma^{2} v \cos v}{\alpha \nu \sin \mu+\gamma \nu \cos v-\left(2 v^{2}-\gamma\right) \sin v} .
$$

\section{Results}

Equation (16) is the main result of this paper. It denotes the relationship between rotation, compression and torsion and generates stability boundaries for the system. When $\alpha$ tends to zero, we have $\mu=0, v=\sqrt{\gamma}$ and so we obtain Wang's result [1], that is, the case of a two-dimensional problem investigating a compressed, rotating beam in the absence of torsion

$$
\chi=-\frac{\gamma \sqrt{\gamma} \cos \sqrt{\gamma}}{\sqrt{\gamma} \cos \sqrt{\gamma}-\sin \sqrt{\gamma}}
$$

shown in Fig. 3a. The stability boundaries are slightly curved. Boundary 1 is asymptotic to the line $\chi=-\gamma$ and boundaries 2, 3 are asymptotic to the values of $\gamma_{2}=20.191$ and $\gamma_{3}=59.679$, that are obtained from Eq. (17), when $\chi$ tends to infinity, that is

$$
\sqrt{\gamma} \cos \sqrt{\gamma}=\sin \sqrt{\gamma}
$$



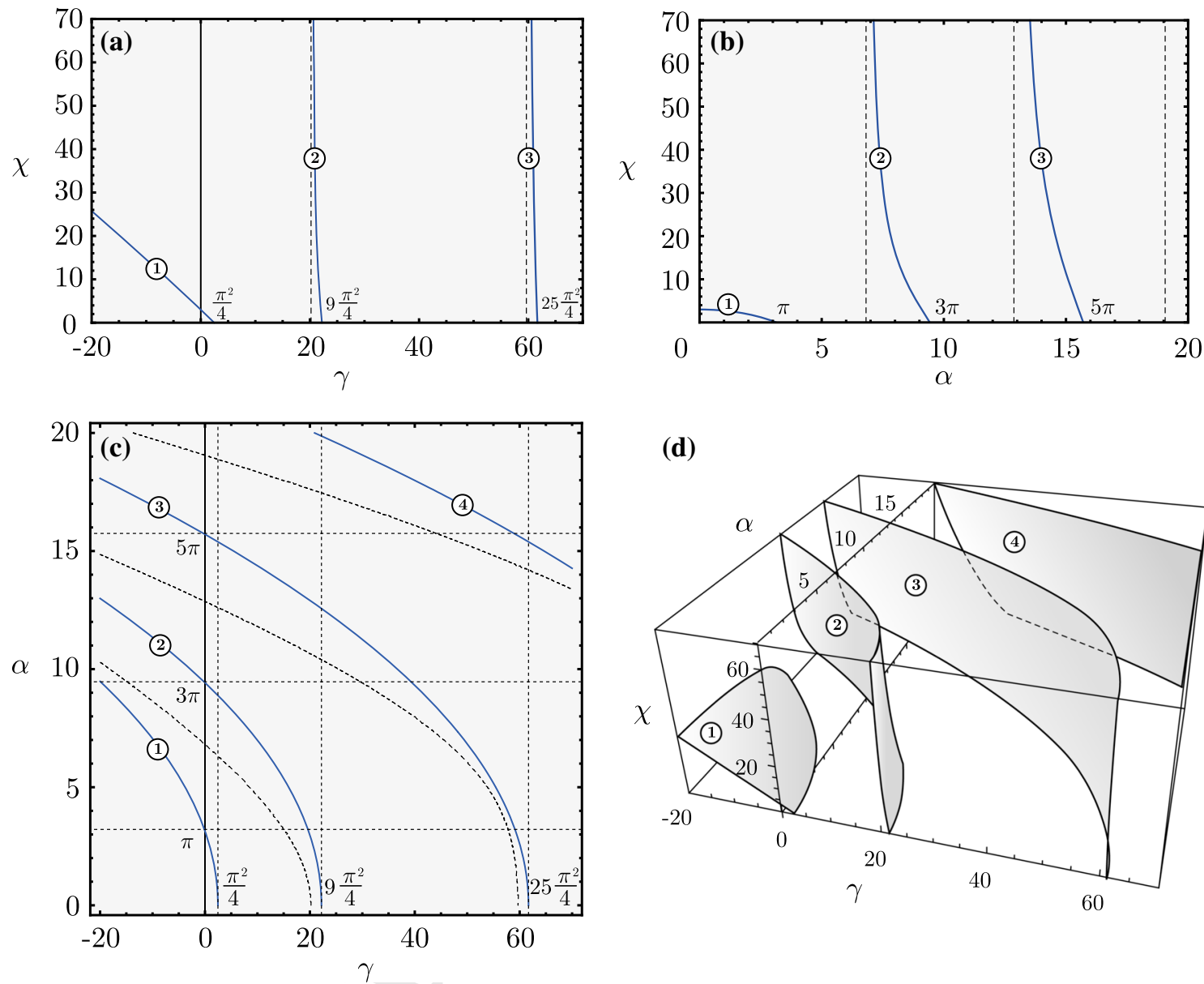

(d)

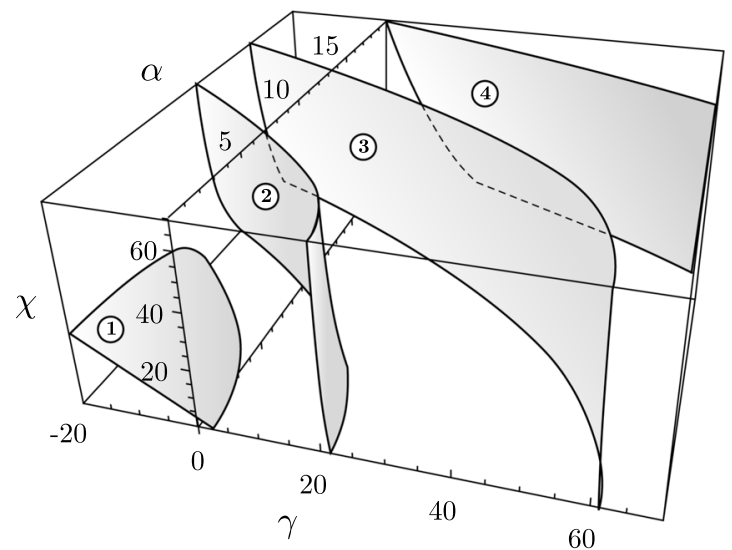

Fig. 3 a Stability boundaries in $(\chi-\gamma)$ plane. b Stability boundaries in $(\chi-\alpha)$ plane. c Stability boundaries in $(\alpha-\gamma)$ plane. d 3D stability boundaries

When $\gamma$ tends to zero $(\alpha \neq 0)$, that is, the relative importance of gravity is negligible, we have that $\mu=v=\alpha / 2$ and hence from Eq. (16) by using L'Hospital's rule

$$
\chi=\frac{\alpha^{3}}{2\left(1+\mu^{2}\right) \tan \mu-\alpha},
$$

the stability boundaries can be seen in Fig. 3b. Boundary 1 tends to the line $\chi=3$ and boundaries are asymptotic to the values of $\alpha_{2}=6.811$ and $\alpha_{3}=12.868$, obtained from Eq. (18), when $\chi$ tends to infinity, that is

$$
2\left(1+\mu^{2}\right) \tan \mu=\alpha .
$$

When $\chi$ might be neglected, the stability boundaries can be seen in Fig. 3c. They are parabolic. The roots along the $\gamma$ axis are the well-known, normalised Euler buckling modes $(2 n+1)^{2} \pi^{2} / 4$ where $n=0,1 \ldots$, and along the $\alpha$ axis are the normalised critical loads in terms of semi-tangential torque $(2 n+1) \pi$ where $n=0,1 \ldots$.

The stability boundaries of the system, corresponding to Eq. (16) are depicted by Fig. 3d. The curvature of the stability boundaries is continuously increasing as the parameter $\alpha$ grows. They are asymptotic to the dashed lines. Looking again at Fig. 3a, surface 1 crosses the $\chi$ axis at $\chi=3$ (see Fig. 4). The system is absolutely stable as long as the solution does not reach the surface 1 . Between surface 1 and 2 instability of the first mode might occur depending on whether the beam stands upward or downward. To the right of surface 2, 3 and 4 the instabilities of the second, third and fourth modes may occur. The validity of the stable and unstable modes is affected by torsion because it bends the stability boundaries as we can see in Fig. 3c. 


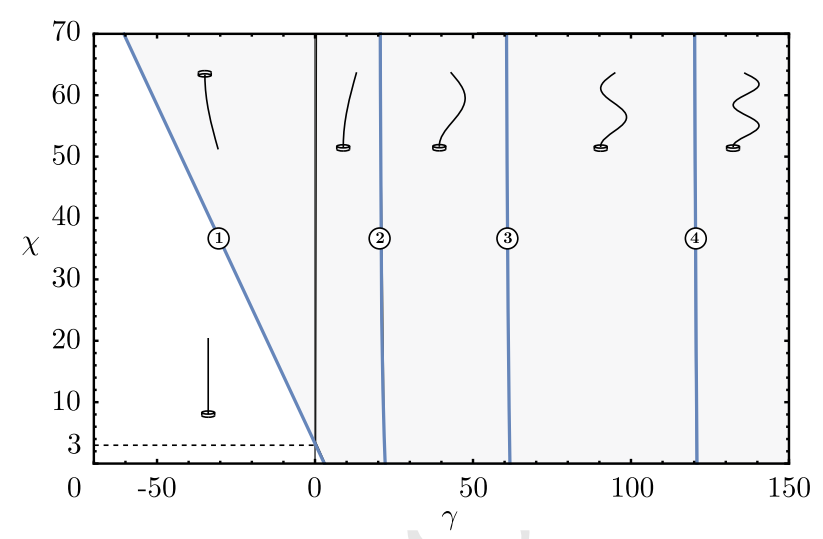

Fig. 4 Explanation of stable and unstable modes

\section{Conclusion}

The paper investigates the structural stability of a light rotating beam under torsion and compression. Torsional torque is assumed to be semi-tangential, and compression is manifested by a block attached to the free end of the shaft. The study is the generalisation of the result of Wang [1] on the stability of a light rotating column with and end mass when torsion is also applied.

There are many advanced practical models in the literature where our analytical results could be applied and extended further: Lee [7], Chen and Liao [8] have investigated the buckling and dynamics stability of a spinning pre-twisted beam under compressive loads. They have taken into account how the speed of rotation affects the stability behaviour of the shaft depending on whether the shaft is pre-twisted or nonpre-twisted. Chen and Peng [9] have examined the dynamic behaviour of a rotating composite beam subjected to axial periodic forces. Como [10] dealt with the case of lateral buckling of a cantilever bar subjected to a transverse following force and studied the stability of bending-torsional equilibrium. Another related topics is vibrations of drill strings where the stability issues have been investigated, for example, by Gulyaev et al. [11] and Liu et al. [12]. Liu et al. [12] presented a discrete system model to study the axial torsional dynamics of a drill string. They also took into account nonlinearities that come from dry friction and loss of contact.

The analytical results of the present paper provide test examples for checking the numerical methods developed for analysis of the above-mentioned mechanical models of the literature.

Acknowledgements The research leading to these results has received funding from the European Research Council under the European Union's Seventh Framework Programme (FP/2007-2013)/ERC Advanced Grant Agreement No. 340889.

\section{References}

1. Wang, C.Y.: Stability of a light rotating column with an end mass. Acta Mech. 43,137-139 (1982). doi:10.1007/BF01175822

2. Timoshenko, S.P., Gere, J.M.: Theory of Elastic Stability, 2nd edn. McGraw-Hill, New York (1963)

3. Ziegler, H.: Principles of Structural Stability, 2nd edn. Birkhauser, Basel (1977). doi:10.1007/978-3-0348-5912-7

4. Beck, M.: Knickung Gerader Stabe Durch Druck und Konservative Torsion. Springer, Berlin (1955)

5. Greenhill, A.G.: On the strength of shafting when exposed both to torsion and to end thrust. Proc. Inst. Mech. Eng. 34, 182-225 (1883). doi:10.1243/PIME-PROC-1883-034-013-02

6. Bazănt, Z., Cedolin, L.: Stability of Structures, pp. 3-8, 46-48. World Scientific Publishing Co. Pte. Ltd. Singapore (2010)

7. Lee, H.P.: Buckling and dynamic stability of spinning pre-twisted beams under compressive axial loads. Int. J. Mech. Sci. 36, 1011-1026 (1994). doi:10.1016/0020-7403(94)90024-8

8. Chen, M.L., Liao, Y.S.: Vibrations of pretwisted spinning beams under axial compressive loads with elastic constraints. J. Sound Vib. 147, 215-230 (1991). doi:10.1016/0022-460X(91)90497-8

9. Chen, L.W., Peng, W.K.: Dynamic stability of rotating composite shafts under periodic axial compressive loads. J. Sound Vib. 212, 497-513 (1998). doi:10.1006/jsvi.1997.1405

10. Como, M.: Lateral buckling of a cantilever subjected to a transverse follower force. Int. J. Solids Struct. 2, 515-523 (1966). doi:10.1016/0020-7683(66)90035-7

11. Gulyaev, V.I., Lugovoi, P.Z., Belova, M.A., Solov'ev, I.L.: Stability of the equilibrium of rotating drillstrings. Int. Appl. Mech. 42, 692-698 (2006). doi:10.1007/s10778-006-0137-5

12. Liu, X., Vlajic, N., Long, X., Meng, G., Balachandran, B.: State-dependent delay influenced drill-string oscillations and stability analysis. J. Vib. Acoust. 136, 051008 (2014). doi:10.1115/1.4027958 